\title{
The equivalence between two-person symmetric games and decision problems
}

Citation for published version (APA):

Ismail, M. S. (2014). The equivalence between two-person symmetric games and decision problems. Maastricht University, Graduate School of Business and Economics. GSBE Research Memoranda No. 023 https://doi.org/10.26481/umagsb.2014023

Document status and date:

Published: 01/01/2014

DOI:

10.26481/umagsb.2014023

Document Version:

Publisher's PDF, also known as Version of record

\section{Please check the document version of this publication:}

- A submitted manuscript is the version of the article upon submission and before peer-review. There can be important differences between the submitted version and the official published version of record.

People interested in the research are advised to contact the author for the final version of the publication, or visit the DOI to the publisher's website.

- The final author version and the galley proof are versions of the publication after peer review.

- The final published version features the final layout of the paper including the volume, issue and page numbers.

Link to publication

\footnotetext{
General rights rights.

- You may freely distribute the URL identifying the publication in the public portal. please follow below link for the End User Agreement:

www.umlib.nl/taverne-license

Take down policy

If you believe that this document breaches copyright please contact us at:

repository@maastrichtuniversity.nl

providing details and we will investigate your claim.
}

Copyright and moral rights for the publications made accessible in the public portal are retained by the authors and/or other copyright owners and it is a condition of accessing publications that users recognise and abide by the legal requirements associated with these

- Users may download and print one copy of any publication from the public portal for the purpose of private study or research.

- You may not further distribute the material or use it for any profit-making activity or commercial gain

If the publication is distributed under the terms of Article $25 \mathrm{fa}$ of the Dutch Copyright Act, indicated by the "Taverne" license above, 


\section{Maastricht University}

Mehmet Ismail

The equivalence between two-person symmetric games and decision problems

RM/14/023

\section{GSBE}

Maastricht University School of Business and Economics

Graduate School of Business and Economics

P.O Box 616

NL- 6200 MD Maastricht

The Netherlands 


\title{
The equivalence between two-person symmetric games and decision problems*
}

\author{
Mehmet ISMAIL ${ }^{\dagger}$ \\ June, 2014
}

\begin{abstract}
We illustrate an equivalence between the class of two-person symmetric games and the class of decision problems with a complete preference relation. Moreover, we show that a strategy is an optimal threat strategy (Nash, 1953) in a two-person symmetric game if and only if it is a maximal element in its equivalent decision problem. In particular, a Nash equilibrium in a two-person symmetric zero-sum game and a pair of maximal elements in its equivalent decision problem coincide. In addition, we show that a two-person symmetric zero-sum game can be extended to its von Neumann-Morgenstern (vN-M) mixed extension if and only if the extended decision problem satisfies the SSB utility (Fishburn, 1982) axioms. Furthermore, we demonstrate that a decision problem satisfies $\mathrm{vN}-\mathrm{M}$ utility if and only if its equivalent symmetric game is a potential game. Accordingly, we provide a formula for the number of linearly independent equations in order for the independence axiom to be satisfied which grows quadratically as the number of alternatives increase.
\end{abstract}

JEL-Classification: C72, D8

*This paper is built upon my master thesis written at University of Paris-1 Panthéon Sorbonne. I thank Itzhak Gilboa for his comments on an early draft. I am also thankful to Jean-Jacques Herings for his feedback. I am particularly indebted to Ronald Peeters for his continuous comments and suggestions. Finally, I thank seminar audience at Maastricht University, Copenhagen University, theoretical and empirical aspects of decision making conference in Bristol 2012, SING9 conference in Vigo and to the audience of poster presentation at the 24th Jerusalem School in Economic Theory. Of course, any mistake is mine.

${ }^{\dagger}$ Maastricht University, e-mail: mehmet@mehmetismail.com 


\section{Introduction}

Relating strategic games and decision problems is not a new idea. For example, Rosenthal (1981) argues that games should be treated as one-person decision problems. By contrast, Fudenberg and Levine (2006) argue that some decision problems can be treated as a game between two selves of a decision maker. The current paper, in distinction, makes a comparison between 'existing' notions between two-person symmetric games and one-person decision problems and shows some equivalences between those notions.

We consider a decision problem consisting of a set of alternatives and a complete (not necessarily transitive) preference relation defined on this set. We show that the class of such decision problems and the class of twoperson symmetric games are equivalent. Accordingly, we consider an identity mapping between those classes. The image of a two-person symmetric game under the mapping is called the equivalent decision problem of the game and the pre-image of a decision problem is called the equivalent game of the decision problem. This equivalence can be interpreted as if the decision maker is playing a game against her dual-self. If the game is of zero-sum then the preferences of the two selves are opposing but this is not necessarily the case if the game is symmetric.

In Section 2, we introduce the framework that will be used throughout the paper. In Section 3, we consider two-person symmetric games in pure strategies and decision problems in a riskless context. We find that a strategy is an optimal pure threat strategy (Nash, 1953) in a two-person symmetric game if and only if it is a maximal element in its equivalent decision problem. In particular, a pure Nash equilibrium in a two-person symmetric zero-sum game and a pair of maximal elements in its equivalent decision problem coincide.

If there does not exist a Nash equilibrium in pure strategies, we usually extend the game in mixed strategies and find a solution there. Similarly, if there is no maximal element in a decision problem we can extend the set of alternatives so as to allow the decision maker to choose a simple lottery. ${ }^{1}$ In Section 4, we extend the games to its von Neumann-Morgenstern (vN-M) mixed extension and we extend the decision problems in such a way that we allow the decision maker to choose simple lotteries over the set of alternatives. We show that a two-person symmetric zero-sum game can be extended to

\footnotetext{
${ }^{1}$ See, for example, Fishburn (1988) for more discussion.
} 
its vN-M mixed extension if and only if the SSB utility (Fishburn, 1982) axioms continuity, dominance and symmetry are satisfied in its equivalent decision problem. Moreover, we show that a mixed strategy is optimal (von Neumann, 1928) in a two-person symmetric zero-sum game if and only if it is a maximal element in its equivalent decision problem. Regarding a twoperson symmetric game in mixed extension, if its equivalent decision problem satisfies the SSB utility axioms then we obtain a two-person symmetric game which is unique up to payoff differences. In addition, a strategy is an optimal mixed threat strategy in a two-person symmetric game if and only if it is a maximal element in its equivalent decision problem defined over simple lotteries.

Finally, in Section 5, we show that a two-person symmetric game is a potential game if and only if its equivalent decision problem satisfies vN-M utility. $^{2}$ Accordingly, we provide the number of linearly independent equations in order for the independence axiom to be satisfied. The number grows quadratically as the number of alternatives increase. This may be interpreted as an alternative way to show the strength of the independence axiom.

\section{The framework}

Firstly, we fix a notation for two-person symmetric games and we define a decision problem for a decision maker. Next, we elaborate on the way we can obtain an equivalence between those two classes.

A two-person non-cooperative game $\left(\{1,2\}, X_{1}, X_{2}, u_{1}, u_{2}\right)$ where $u_{1}, u_{2}$ : $X_{1} \times X_{2} \rightarrow \mathbb{R}$ is called symmetric if $X_{1}=X_{2}$ and $u_{1}(x, y)=u_{2}(y, x)$ for all $x, y$ in $X$. We denote a two-person symmetric game by $(X, u)$ where both players have the same arbitrary set of pure actions $X$ and $u: X \times X \rightarrow \mathbb{R}$. When player 1 plays $x$ and player 2 plays $y$ player 1 receives $u(x, y)$ and player 2 receives $u(y, x)$. We use the notation $(X, v)$ to represent a twoperson symmetric zero-sum game, i.e. $v(x, y)+v(y, x)=0$ for all $x, y$ in $X$.

We call the pair $(X, \succeq)$ a decision problem where $X$ is a set of alternatives available to a decision maker. The relation $\succeq \subseteq X \times X$ represents the

\footnotetext{
${ }^{2}$ This is not the first example of relating vN-M utility with a game theoretical concept. For instance, Roth (1977) shows that Shapley value of a player and vN-M utility function coincide if we assume the player's preferences over the positions can be represented by that utility function and those preferences satisfy some assumptions.
} 
preferences of the decision maker over $X$. It can be read as weakly preferred or at least as good as. The indifference part of $\succeq$ is defined as usual: We write $x \sim y$ if $x \succeq y$ and $y \succeq x$. Moreover, we write $x \succ y$ if $x \succeq y$ but not $x \sim y$. One can interpret the decision problem $(X, \succeq)$ as if a decision maker wants to choose an element from $X$ taking into account her preferences.

If we assume that a preference relation on a countable set is reflexive, complete and transitive then it is representable by a one-variable order-preserving utility function. ${ }^{3}$ However, this is not possible if we remove the transitivity assumption. In that case, a simple but powerful approach to obtain a representation is to consider a two-variable utility function. ${ }^{4}$ The following proposition states that such a decision problem is representable.

Proposition 1. If the relation $\succeq$ on a set $X$ is complete. Then there exist $U: X \times X \rightarrow \mathbb{R}$ such that for all $x, y$ in $X, x \succ y \Leftrightarrow U(x, y)>U(y, x)$.

Proof. Let $\succeq$ be a complete relation on $X$. Define a real valued function $U$ such that for all $(x, y) \in X \times X$ with $x \succ y$ we have $U(x, y)>U(y, x)$. In addition, for all $x \sim y$ define $U(x, y)=U(y, x)$. It is left to show that $U(z, w)>U(w, z)$ for $z, w$ in $X$ implies that $z \succ w$. Assume that $z \preceq w$, then $U(z, w) \leq U(w, z)$ which is a contradiction to our supposition.

Remark 1. Let $U: X \times X \rightarrow \mathbb{R}$ be a function. Then the preference relation on $X$ defined by $x \succ y$ if $U(x, y)>U(y, x)$ and $x \sim y$ if $U(x, y)=U(y, x)$ would be complete and such $U$ would represent $\succeq$.

The function $U(x, y)$ may be (loosely) interpreted as a magnitude of the intensity of preference for $x$ over $y$. If the intensity of preference for $x$ over $y$ is larger than the one for $y$ over $x$, then we say $x$ is preferred to $y$. In particular, for $x, y$ in $X$ the difference $U(x, y)-U(y, x)$ defines another function, say $V(x, y)$, which has a nice property of being skew-symmetric, i.e. $V(x, y)=$ $-V(y, x)$. The following corollary illustrates this situation.

Corollary 1. If the relation $\succeq$ on a set $X$ is complete. Then there exist $V: X \times X \rightarrow \mathbb{R}$ such that for all $x, y \in X, x \succ y \Leftrightarrow V(x, y)>0$ with $V(x, y)+V(y, x)=0$.

\footnotetext{
${ }^{3}$ See, for example, Debreu (1954).

${ }^{4}$ One of the most well-known supporter of this approach is probably Fishburn. Fishburn (1982) generalized the expected utility of von Neumann and Morgenstern by a skewsymmetric two-variable utility function without using the transitivity and the independence axioms.
} 
We call the triplets $(X, U, \succeq)$ and $(X, V, \succeq)$ a decision problem as well. Notice that a function $U$ as in Remark 1 induces a unique preference relation on $X$ hence $(X, U, \succeq)$ is a more specific decision problem than $(X, \succeq)$. We will write $(X, U)$ instead of $(X, U, \succeq)$ and each time we refer to a decision problem we will state which form we consider. Note that given a decision problem $(X, \succeq)$ there are (uncountably) many functions $U$ that represent $\succeq$. Given a decision problem $(X, \succeq)$ we define the set

$$
[\succeq]=\{U: X \times X \rightarrow \mathbb{R} \mid U \text { represents } \succeq\} .
$$

It will be helpful in order to identify the similarities of the functions representing the same relation.

\section{Games in pure strategies and decision prob- lems in a riskless context.}

\subsection{The equivalence mapping.}

Let $\mathcal{D}$ denote the class of all decision problems that can be written as $(X, U)$ where $U$ is an order-preserving utility function as in Proposition 1 and let $\mathcal{G}$ denote the class of all two-person symmetric games. The next proposition shows the equivalence between those two classes. We will make use of it to derive conclusions for elements in one class with the help of the structure of the elements in the other class.

Proposition 2. $\mathcal{G}=\mathcal{D}$.

Proof. To show $\mathcal{G} \subseteq \mathcal{D}$, let $(X, u)$ be a game in $\mathcal{G}$. Since $u: X \times X \rightarrow \mathbb{R}$, by Remark 1 the preference relation defined by $x \succ y$ if $u(x, y)>u(y, x)$ would be complete and $u$ represents $\succeq$. Accordingly, $(X, u, \succeq)$ belongs to the desired class when we consider $X$ as the set of alternatives. Conversely, given a decision problem $(X, U)$, we define the two-person symmetric game as follows. We consider $X$ as the set of pure strategies for each player and when player 1 plays $x \in X$ and player 2 plays $y \in X$, player 1 receives a payoff of $U(x, y)$ and player 2 receives $U(y, x)$. Consequently, the game $(X, U)$ is a two-person symmetric game.

In particular, the class of decision problems with a representation function $V$ as in Corollary 1 is equivalent to the class of two-person symmetric zero- 


\begin{tabular}{c|c|c|c|}
\multicolumn{1}{c}{} & \multicolumn{1}{c}{$\mathrm{x}$} & \multicolumn{1}{c}{$\mathrm{y}$} & \multicolumn{1}{c}{$\mathrm{z}$} \\
\cline { 2 - 4 } $\mathrm{x}$ & 1,1 & 3,0 & 0,3 \\
\cline { 2 - 4 } $\mathrm{y}$ & 0,3 & 1,1 & 3,0 \\
\cline { 2 - 4 } $\mathrm{z}$ & 3,0 & 0,3 & 1,1 \\
\cline { 2 - 4 } & & &
\end{tabular}

\begin{tabular}{c|c|c|c|}
\multicolumn{1}{c}{$\mathrm{x}$} & \multicolumn{1}{c}{$\mathrm{y}$} & \multicolumn{1}{c}{$\mathrm{z}$} \\
\cline { 2 - 4 } $\mathrm{x}$ & 0,0 & $1,-1$ & $-1,1$ \\
\cline { 2 - 4 } $\mathrm{y}$ & $-1,1$ & 0,0 & $1,-1$ \\
\cline { 2 - 4 } $\mathrm{z}$ & $1,-1$ & $-1,1$ & 0,0 \\
\cline { 2 - 4 } & &
\end{tabular}

Figure 1: Games $(X, u)$ and $(X, v)$ respectively.

sum games. Since the construction is essentially the same with the preceding proposition we omit it.

So far, we considered the pair $(X, u)$ both as a game and as a decision problem. To stress this, we define an identity mapping $\mathcal{T}: \mathcal{G} \rightarrow \mathcal{D}$. That is, the mapping $\mathcal{T}$ does not change the mathematical object but it puts a different interpretation on it. In other words, if we interpret a given pair $(X, u)$ as a two-person symmetric game $\left(\{1,2\}, X, X, u, u^{\top}\right)$ then we interpret the image of $(X, u)$ under $\mathcal{T}$ as the decision problem $(X, u, \succeq)$ where $u$ is the order-preserving utility function representing the relation $\succeq$. Note also that the mapping $\mathcal{T}$ is bijective by Proposition 2.

In addition, if $u$ represents the preference relation $\succeq$ on $X$ then we call the pair $(X, \succeq)$ the equivalent decision problem of the two-person symmetric game $(X, u)$. To avoid confusion and to distinguish between decision problems and games, we write $\mathcal{T}((X, u))=(X, U)$ where $u=U$. Then we say the equivalent decision problem of the game $(X, u)$ is $(X, U) .^{5}$

One may informally interpret an equivalent game of a decision problem as follows. Given a decision problem, one may think of the decision maker as if she is playing against her dual-self. ${ }^{6}$ If one considers the equivalent game in the form of two-person symmetric zero-sum, then what the decision maker gains is the loss of her dual-self. This is, however, not necessarily the case if one considers the equivalent two-person symmetric game.

Let us give an example for better understanding how decision problems and games in question are related. Figure 1 presents a two-person symmetric game $(X, u)$ and a two-person symmetric zero-sum game $(X, v)$. Consider the equivalent decision problem $(X, U)$ of the game $(X, u)$ under the mapping $\mathcal{T}$ where $U=u$. The preference relation represented by the utility function $U$ is $x \succ y \succ z \succ x$ because $U(x, y)>U(y, x), U(y, z)>U(z, y)$ and

\footnotetext{
${ }^{5}$ Those notations will be helpful because most of the time we will need to make distinctions between the decision problems of the form $(X, U),(X, V)$ and $(X, \succeq)$.

${ }^{6}$ See Fudenberg and Levine (2006) for a similar interpretation of decision problems.
} 
$U(z, x)>U(x, z)$. Similarly, consider the equivalent decision problem $(X, V)$ of the game $(X, v)$ under the mapping $\mathcal{T}$ where $V=v$. Since $V(x, y)>0$, $V(y, z)>0$ and $V(z, x)>0$, the preference relation turns out to be the same as before, i.e. $x \succ y \succ z \succ x$. Therefore, we can argue that both games have the same decision problem (in a general sense) $(X, \succeq)$ and that both $u$ and $v$ represent $\succeq$, that is $u$ and $v$ in $[\succeq]$. In the following section, we will show that different games whose equivalent decision problems have the same preferences share some common properties.

Notice that the game $(X, v)$ in Figure 1 is the classical rock-paper-scissors game. This game is known by its cyclic structure. In other words, the relation defined by 'one shape beats the other' is cyclic because rock beats scissors, scissors beats paper and paper beats rock. Intuitively speaking, it would be expected that the equivalent decision problem (if ever exists) of rock-paperscissors game should have cyclic preferences which is actually the case as we show above.

\subsection{Counterparts of maximal elements.}

We say that a set $X$ admits a maximal element $x$ with respect to a preference relation $\succeq$ on $X$ if there exists no element $y \in X$ such that $y \succ x$. If $\succeq$ is complete then one can write $x \succeq y$ for all $y$ in $X$.

The following proposition characterizes the pure Nash equilibria in twoperson symmetric zero-sum games with respect to the maximal elements of decision problems.

Proposition 3. Let $(X, v)$ be a two-person symmetric zero-sum game and let $(X, \succeq)$ be its equivalent decision problem. $X$ admits a maximal element $x^{*}$ with respect to $\succeq$ if and only if the game $(X, v)$ possesses a pure Nash equilibrium $\left(x^{*}, x^{*}\right)$.

Proof. ' $\Rightarrow$ ': Since $(X, \succeq)$ is the equivalent decision problem of the game $(X, v)$, the function $v$ represents the relation $\succeq$ in the sense of Corollary 1 . Hence $x^{*} \succeq y$ if and only if $v\left(x^{*}, y\right) \geq 0$ for all $y \in X$. Since $(X, v)$ is a two-person symmetric zero-sum game if there is a value it should be zero. Since $x^{*}$ guarantees the value 0 it is an optimal strategy. By symmetry, the pair $\left(x^{*}, x^{*}\right)$ is a pure Nash equilibrium of $(X, v)$.

' $\Leftarrow$ ': If $\left(x^{*}, x^{*}\right)$ is an equilibrium of the game $(X, v)$, then the value of the game exists and it is 0 . Moreover $x^{*}$ is an optimal strategy and it guarantees 
the value 0 , that is $v\left(x^{*}, y\right) \geq 0$ for all $y \in X$. It implies that $x^{*} \succeq y$ for all $y \in X$ since $v$ represents the relation $\succeq$. Hence $x^{*}$ is a maximal element.

In two-person symmetric games, it turns out that a maximal element coincides with an optimal threat strategy that we know from Nash (1953) bargaining problem. A strategy is called an optimal threat strategy in a twoperson game if it is an optimal strategy of the game which is obtained from the payoff differences of the original game. ${ }^{7}$ In addition, an evolutionary stable strategy for finite populations (Schaffer, 1988) coincides with an optimal pure threat strategy in two-person symmetric games if the population consists of two individuals and the contest size is two as well.

The following proposition illustrates the relationship between an optimal pure threat strategy and a maximal element of a decision problem.

Proposition 4. Let $(X, u)$ be a two-person symmetric game and let $(X, \succeq)$ be its equivalent decision problem. An element $x^{*} \in X$ is maximal with respect to $\succeq$ if and only if $x^{*}$ is an optimal pure threat strategy in $(X, u)$.

Proof. Since $(X, \succeq)$ is the equivalent decision problem of $(X, u)$, the function $u$ represents the relation $\succeq$. So $x^{*}$ is maximal with respect to $\succeq$ if and only if $u\left(x^{*}, y\right) \geq u\left(y, x^{*}\right)$ for all $y \in X$. It is equivalent to $u\left(x^{*}, y\right)-u\left(y, x^{*}\right) \geq 0$ for all $y \in X$ which means $x^{*}$ is an optimal strategy in the game derived from the payoff differences of the game $(X, u)$. That is, $x^{*}$ is an optimal threat strategy in $(X, u)$.

To elaborate on Proposition 3 and Proposition 4 a little bit more, consider an arbitrary decision problem $(X, \succeq)$. If $X$ admits a maximal element with respect to $\succeq$ then all two-person symmetric games $(X, u)$ with $u \in[\succeq]$ have an optimal pure threat strategy and all two-person symmetric zero-sum games $(X, v)$ with $v \in[\succeq]$ have a pure Nash equilibrium. The reason is that both $u$ and $v$ represent the same preference relation $\succeq$. In addition, the reverse implications are true as well.

\footnotetext{
${ }^{7}$ Note that here we use the definition of optimal threat strategy for transferable utility games. See, for example, Owen (1968) for a detailed discussion.
} 


\section{Mixed extension of games and of decision problems}

So far we have considered the games with arbitrary pure strategy sets. We elaborate on the mixed extension of finite games in this section. If a game has no Nash equilibrium in pure strategies then we usually consider the game in mixed extension. That is, players are allowed to randomize over the pure strategies. In the case of finite games, the problem of existence of a Nash equilibrium is solved in this way. Similarly, we can think of randomization in decision problems in case there is no maximal element.

Let $(X=\{x, y, z\}, \succeq)$ be a decision problem such that $x \succ y \succ z \succ x$. Then a paradoxical situation arises according to Tullock (1964) when a decision maker is to choose an alternative from $X$. One way to solve this problem is to allow the decision maker to randomize over the set of alternatives. $^{8}$ Remember the rock-paper-scissors game in Figure 1. The unique Nash equilibrium is to play each action with equal probability. As we showed, the equivalent decision problem of rock-paper-scissors game is $(X=\{x, y, z\}, \succeq)$ where $\succeq$ is cyclic. Therefore, one may suspect that the solution to the decision problem $(X=\{x, y, z\}, \succeq)$ is to choose each alternative with equal probability as well. Formally, we consider the extension of decision problems to the set of simple probability distributions over the set of alternatives.

\subsection{Two-player symmetric zero-sum games}

The usual mixed extension of a finite two-person symmetric zero-sum game $(X, v)$ is considered to be the game $\left(\Delta(X), v^{\prime}\right)$ where the von NeumannMorgenstern utility function $v^{\prime}$ is the bilinear extension of $v$ to $\Delta(X) \times \Delta(X)$.

Now consider a finite decision problem $(X, V)$. We extend the function $V$ on $X \times X$ to a function $V^{\prime}$ on $\Delta(X) \times \Delta(X)$ such that $V_{\mid X \times X}^{\prime}=V$. Accordingly, the preference relation $\succeq$ on $X$ is also extended to $\succeq^{\prime}$ on $\Delta(X)$ so that $\succeq_{\mid X \times X}^{\prime}=\succeq$. We denote the mixed extension of a decision problem as $\left(\Delta(X), V^{\prime}, \succeq^{\prime}\right)$ or shortly $\left(\Delta(X), V^{\prime}\right)$.

It turns out that there is a connection with the extended preference relation and the SSB (skew-symmetric and bilinear) utility theory of Fishburn (1982). This theory is based on the following axioms: For all $p, q$ and $r$ in $\Delta(X)$

\footnotetext{
${ }^{8}$ See, for example, Fishburn (1988, p. 137).
} 
Axiom $\mathbf{C}$ (Continuity). There exists $\beta \in(0,1)$ such that $p \succ q \succ r$ implies $q \sim \beta p+(1-\beta) r$.

Axiom D (Dominance). For all $\alpha \in(0,1), p \succ q$ and $p \succeq r$ implies $p \succ$ $\alpha q+(1-\alpha) r, q \succ p$ and $r \succeq p$ implies $\alpha q+(1-\alpha) r \succ p$, and $p \sim q$ and $p \sim r$ implies $p \sim \alpha q+(1-\alpha) r$.

Axiom S (Symmetry). For all $\alpha \in(0,1), p \succ q \succ r, p \succ r$ and $q \sim \frac{1}{2} p+\frac{1}{2} r$ implies $\alpha p+(1-\alpha) r \sim \frac{1}{2} p+\frac{1}{2} q \Leftrightarrow \alpha r+(1-\alpha) p \sim \frac{1}{2} r+\frac{1}{2} q$.

As the following theorem states, those axioms are necessary and sufficient for representing the preferences by an SSB utility function.

Theorem (Fishburn, 1982). The relation $\succeq$ on $\Delta(X)$ satisfies $\mathrm{C}, \mathrm{D}$ and $\mathrm{S}$ if and only if there exists an SSB function $\phi$ such that $p \succ q \Leftrightarrow \phi(p, q)>0$ for all $p, q$ in $\Delta(X)$. Moreover, $\phi$ is unique up to multiplication by a positive constant.

Let $\mathcal{G}^{\prime} \subset \mathcal{G}$ be the class of two-person symmetric zero-sum games in vN-M mixed extension $\left(\Delta(X), v^{\prime}\right)$ and let $\mathcal{D}^{\prime} \subset \mathcal{D}$ be the class of decision problems of the form $\left(\Delta(X), V^{\prime}\right)$. Consider again the identity mapping $\mathcal{T}$ whose domain and codomain are restricted to $\mathcal{G}^{\prime}$ and $\mathcal{D}^{\prime}$ respectively. Note that mapping $\mathcal{T}$ is one-to-one but not onto because $\mathcal{G}^{\prime} \subset \mathcal{D}^{\prime}$. For instance, there could be decision problems in $\mathcal{D}^{\prime}$ whose representation function does not satisfy bilinearity. To obtain a bijective mapping we need to put more assumptions on the preferences of decision problems in $\mathcal{D}^{\prime}$. Similar as before, we say that a decision problem $\left(\Delta(X), \succeq^{\prime}\right)$ is the equivalent decision problem of a two-person symmetric zero-sum game $\left(\Delta(X), v^{\prime}\right)$ if the utility function $v^{\prime}$ under mapping $\mathcal{T}$ happens to represent the relation $\succeq^{\prime}$.

The following proposition characterizes the vN-M mixed extension of a game with the properties of its equivalent decision problem.

Proposition 5. Let $(X, v)$ be a two-person symmetric zero-sum game and let $(X, \succeq)$ be its equivalent decision problem under $\mathcal{T} .(X, v)$ can be extended to $v N-M$ mixed extension $\left(\Delta(X), v^{\prime}\right)$ if and only if the preference relation $\succeq^{\prime}$ of the extended decision problem $\left(\Delta(X), \succeq^{\prime}\right)$ satisfies the axioms $C, D$ and $S$.

Proof. ' $\Rightarrow$ ': Let $\left(\Delta(X), v^{\prime}\right)$ be the equivalent decision problem of the game $\left(\Delta(X), v^{\prime}\right)$. We show that $v^{\prime}$ is skew-symmetric and bilinear and hence it satisfies the axioms $C, D$ and $S$ by the theorem of Fishburn (1982). The 

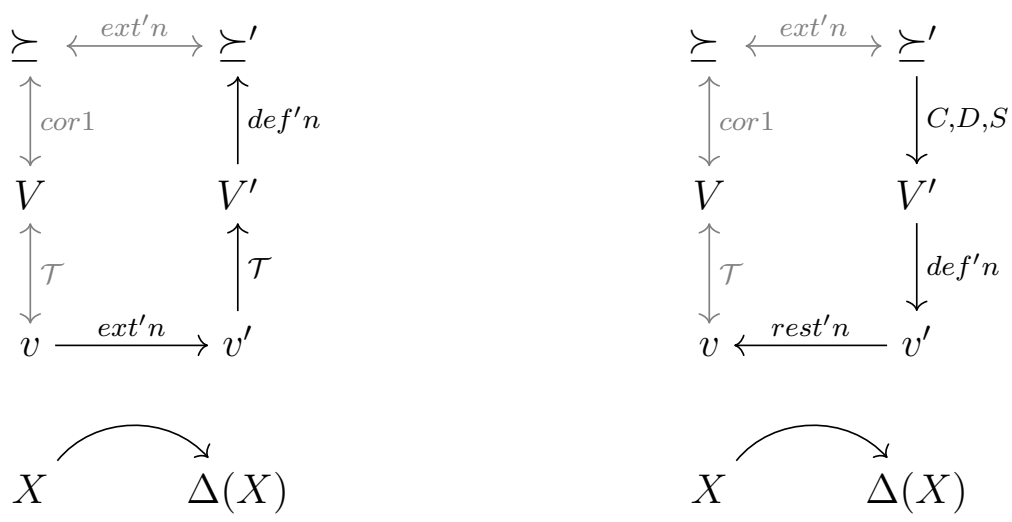

Figure 2: Illustration of Proposition 5.

function $v^{\prime}$ is skew-symmetric, i.e. $v^{\prime}(p, q)=-v^{\prime}(q, p)$ for all $p, q \in \Delta(X)$ since $v^{\prime}(p, q)=p^{\top} A q$ where $A$ is the payoff matrix of player 1 with $A=-A^{\top}$. Then $p^{\top} A q=p^{\top}\left(-A^{\top}\right) q=\left(p^{\top}\left(-A^{\top}\right) q\right)^{\top}=-q^{\top} A p=-v^{\prime}(q, p)$. The payoff function $v^{\prime}$ is bilinear by definition.

' $\Leftarrow$ ': We extend the decision problem $(X, \succeq)$ to $\left(\Delta(X), \succeq^{\prime}\right)$. If the relation $\succeq^{\prime}$ satisfies the axioms $C, D$ and $S$ then by Fishburn (1982) there exist a skew-symmetric bilinear function $\phi$ which is unique up to a multiplication by a positive constant such that $p \succ q \Leftrightarrow \phi(p, q)>0$ where $\phi(p, q)=$ $\sum_{x \in X} \sum_{y \in X} p(x) q(y) \phi(x, y)$ for all $p, q$ in $\Delta(X)$. Since $\phi$ is the extension of $v$, i.e. $\phi(x, y)=v(x, y)$ for all $x, y$ in $X$, we obtain a unique $\phi$ on $\Delta(X)$ which represents $\succeq^{\prime}$. Define the payoff function $v^{\prime}$ of player 1 as $v^{\prime}(p, q)=\phi(p, q)$ for all $p, q$ in $\Delta(X)$. Consequently, we obtain the mixed extension $\left(\Delta(X), v^{\prime}\right)$ of the two-person symmetric zero-sum game $(X, v)$.

Figure 2 illustrates Proposition 5 and its proof. It basically shows the steps to reach the extended preference relation $\succeq^{\prime}$ on $\Delta(X)$ which satisfies the axioms $C, D$ and $S$ and vice versa. Each arrow and text say what to do in order to pass from one object to another. ${ }^{9}$

\footnotetext{
${ }^{9}$ For example, let us start by moving counter-clockwise from $v$ which is located on the left bottom corner. By considering the vN-M extension of the game $(X, v)$ we can obtain the extended function $v^{\prime}$. Then by applying $\mathcal{T}$ we obtain the extended decision problem $\left(\Delta(X), V^{\prime}\right)$. Accordingly, we define the extended preference relation $\succeq^{\prime}$ from $V^{\prime}$ which is shown by Proposition 5 to satisfy the SSB utility axioms $C, D$ and $S$. Finally, by taking the restriction of $V^{\prime}$ on $X$ we obtain the decision problem $(X, V)$ and hence the non-extended game $(X, v)$.
} 
Now, let $\mathcal{D}^{S S B} \subset \mathcal{D}^{\prime}$ be the class of decision problems $(\Delta(X), v)$ where $v$ is an SSB function. The following corollary shows that the identity mapping $\mathcal{T}$ becomes bijective when we restrict the domain to $\mathcal{G}^{\prime}$ and the codomain to $\mathcal{D}^{S S B}$.

Corollary 2. $\mathcal{G}^{\prime}=\mathcal{D}^{S S B}$.

Regarding the two-person symmetric zero-sum games, the above corollary shows that we can obtain a result similar to Proposition 2 in mixed strategies as well. We also obtain an analogoue of Proposition 3 as the following proposition shows.

Proposition 6. Let $(\Delta(X), v)$ be a two-person symmetric zero-sum game and let $(\Delta(X), \succeq)$ be its equivalent decision problem. Then, a strategy $p$ is optimal if and only if $p$ is a maximal element with respect to $\succeq$.

Proof. ' $\Rightarrow$ ': The value of a two-person symmetric zero-sum game is 0 . Let $p$ be an optimal strategy then $v(p, q) \geq 0$ for all $q \in \Delta(X)$. Since $v$ represents $\succeq$ it implies that $p \succeq q$ for all $q \in \Delta(X)$.

' $\Leftarrow$ ': If $p$ is maximal then $v(p, q) \geq 0$ for all $q \in \Delta(X)$. Since $p$ guarantees the value of the game it is an optimal strategy in $(\Delta(X), v)$.

\subsection{Two-player symmetric games}

Similarly, the mixed extension of a finite two-person symmetric game $(X, u)$ is the game $\left(\Delta(X), u^{\prime}\right)$ where the vN-M utility function $u^{\prime}$ is the bilinear extension of $u$ to $\Delta(X) \times \Delta(X)$. Given a decision problem $(X, U)$ where $X$ is finite, we extend the function $U$ to a function $U^{\prime}$ on $\Delta(X) \times \Delta(X)$ such that $U_{\mid X \times X}^{\prime}=U$. This extends the relation $\succeq$ on $X$ to $\succeq_{\mid X \times X}^{\prime}$ on $\Delta(X)$. Let $\mathcal{G}^{\prime \prime} \subset \mathcal{G}$ denote the class of two-person symmetric games in vN-M mixed extension $(\Delta(X), u)$ and let $\mathcal{D}^{\prime \prime} \subset \mathcal{D}$ denote the class of decision problems of the form $\left(\Delta(X), U^{\prime}\right)$. The following proposition illustrates that the vN-M extension of a symmetric game implies SSB utility in its equivalent decision problem.

Proposition 7. Let $(X, u)$ be a two-person symmetric game and let $(X, \succeq)$ be its equivalent decision problem. If one extends $(X, u)$ to $v N-M$ mixed extension $\left(\Delta(X), u^{\prime}\right)$ then the extended preference relation $\succeq^{\prime}$ on $\Delta(X)$ satisfies the axioms $C, D$ and $S$. 
Proof. Showing that the relation $\succeq^{\prime}$ defined by $p \succeq^{\prime} q \Leftrightarrow u^{\prime}(p, q) \geq u^{\prime}(q, p)$ satisfies $C, D$ and $S$ is equivalent to showing that the function $v(p, q)$ defined by $u(p, q)-u(q, p)$ for all $p, q$ in $\Delta(X)$ is an SSB function. By definition $v$ is a skew-symmetric function. The bilinearity of $v$ follows from the bilinearity of $u$.

Regarding the other direction, if an extended decision problem satisfies SSB utility then it is possible construct a two-person symmetric game which is unique up to payoff differences. That is $u$ and $u^{\prime}$ are such functions if and only if $u(p, q)-u(q, p)=u^{\prime}(p, q)-u^{\prime}(q, p)$ for all $p, q$ in $\Delta(X)$. Accordingly, such a function $u$ becomes $\Delta$-bilinear, that is, the function $u(p, q)-u(q, p)$ is bilinear for all $p$ and $q$ in the domain of $u$. To be more precise, the identity mapping $\mathcal{T}$ restricted to domain $\mathcal{G}^{\prime \prime}$ and to codomain $\mathcal{D}^{\prime \prime}$ is one-to-one but not onto. However, it remains to be an open question which axioms are exactly needed on the extended preferences in order to have that mapping onto. ${ }^{10}$ The following proposition shows these formally.

Proposition 8. Let $(X, u)$ be a two-person symmetric game, let $(X, u, \succeq)$ be its equivalent decision problem and let $\left(\Delta(X), u^{\prime}\right)$ be the $v N-M$ mixed extension of the game. If the preference relation $\succeq^{\prime}$ of the extended decision problem $\left(\Delta(X), U^{\prime}, \succeq^{\prime}\right)$ satisfies the axioms $C, D$ and $S$ then $u^{\prime} \in\left[\succeq^{\prime}\right]$. Moreover, any $U^{\prime}$ in $\left[\succeq^{\prime}\right]$ is unique up to payoff differences and is $\Delta$-bilinear.

Proof. Firstly, we show that $u^{\prime} \in\left[\succeq^{\prime}\right]$. We the function $v^{\prime}$ by $v^{\prime}(p, q)=$ $U^{\prime}(p, q)-U^{\prime}(q, p)$ for all $p, q$ in $\Delta(X)$ with $U^{\prime} \in\left[\succeq^{\prime}\right]$ so that we have $p \succeq^{\prime}$ $q \Leftrightarrow v^{\prime}(p, q) \geq 0$. If we assume that $\succeq^{\prime}$ satisfies the axioms $C, D$ and $S$, then $v^{\prime}$ becomes an SSB function by Theorem 1 of Fishburn (1982). Since $U^{\prime}$ and $u^{\prime}$ are the extensions of $u$, we have $v^{\prime}(x, y)=u(x, y)-u(y, x)=$ $u^{\prime}(x, y)-u^{\prime}(y, x)$ for all $x, y$ in $X$. Since both $v^{\prime}$ and $u^{\prime}$ are bilinear, we have $v^{\prime}(p, q)=u^{\prime}(p, q)-u^{\prime}(q, p)$ for all $p, q$ in $\Delta(X)$. Consequently, we obtain $p \succeq^{\prime} q \Leftrightarrow u^{\prime}(p, q) \geq u^{\prime}(q, p)$, that is $u^{\prime} \in\left[\succeq^{\prime}\right]$.

Secondly, we show that a function $U^{\prime}$ in $\left[\succeq^{\prime}\right]$ is unique up to the difference in payoffs. Observe that $v^{\prime}$ is uniquely defined on the whole domain since the values of $v^{\prime}(x, y)=u(x, y)-u(y, x)$ are fixed for all $x, y$ in $X$. Let $u_{1}$ and $u_{2}$ in $\left[\succeq^{\prime}\right]$, then we obtain $u_{1}(p, q)-u_{1}(q, p)=u_{2}(p, q)-u_{2}(q, p)$ for all $p, q$ in

\footnotetext{
${ }^{10}$ It is actually not obvious whether it is even possible to have a set of axioms on a preference relation such that the representation function $u$ becomes unique (up to a positive linear transformation).
} 
$\Delta(X)$. Finally, we show that any $U^{\prime}$ in $\left[\succeq^{\prime}\right]$ is $\Delta$-bilinear. We have

$$
v^{\prime}(\alpha p+(1-\alpha) r, q)=u_{1}(\alpha p+(1-\alpha) r, q)-u_{1}(q, \alpha p+(1-\alpha) r) .
$$

Then, bilinearity of $v^{\prime}$ implies that,

$\alpha v^{\prime}(p, q)+(1-\alpha) v^{\prime}(r, q)=\alpha u_{1}(p, q)+(1-\alpha) u_{1}(r, q)-\alpha u_{1}(q, p)-(1-\alpha) u_{1}(q, r)$.

The $\Delta$-bilinearity in the second argument is essentially the same hence the proof is complete.

The following proposition illustrates that it is possible to obtain a result in the mixed extension of symmetric games which analogous to Proposition 4 .

Proposition 9. Let $(\Delta(X), u)$ be a two-person symmetric game and let $\left(\Delta(X), \succeq^{\prime}\right)$ be its equivalent decision problem. Then a strategy $p^{*}$ is maximal with respect to $\succeq^{\prime}$ if and only if $p^{*}$ is an optimal threat strategy.

Proof. Since $u$ represents $\succeq^{\prime}$, we have that $p^{*}$ is a maximal element if and only if $u\left(p^{*}, q\right) \geq u\left(q, p^{*}\right)$. Hence $p^{*}$ is an optimal threat strategy in $(\Delta(X), u)$.

Note that for any $u^{\prime} \in\left[\succeq^{\prime}\right]$, the game $\left(\Delta(X), u^{\prime}\right)$ is a two-person symmetric game but not necessarily it is the vN-M mixed extension of the game $\left(X, u^{\prime}\right)$. However, all the games $\left(\Delta(X), u^{\prime}\right)$ with $u^{\prime} \in\left[\succeq^{\prime}\right]$ have the same optimal threat strategy $p^{*}$ because the payoff functions simply represent the same preference relation.

\section{The independence axiom}

The difference between expected utility and SSB utility is that transitivity and the axiom of independence is not assumed in SSB utility. One might wonder what happens to its equivalent game if the decision problem satisfies the independence axiom. In that case, we observe that the equivalent two-person symmetric game becomes a potential game and the game matrix satisfies a sort of triangular equality condition. The following definitions and proposition illustrate this situation in a formal way.

To the best of our knowledge, the subclass of potential games were first proposed by Rosenthal (1973) and later formalized by Monderer and Shapley (1996). It is a useful class because all potential games possess a pure Nash 
equilibrium. Moreover, the potential function can be used for Nash equilibrium selection. ${ }^{11}$ For our purpose here, we use the definition of the potential game for two-person symmetric games.

Definition 1. A symmetric two-person game $(X, u)$ is called a (exact) potential game if there exists a function $P: X \times X \rightarrow \mathbb{R}$ such that for all $y$ and all $x, z$ in $X$, we have $u(x, y)-u(z, y)=P(x, y)-P(z, y)$ and $u(x, y)-u(z, y)=P(y, x)-P(y, z) \cdot{ }^{12}$

Now let us also recall the independence axiom. A preference relation $\succeq$ on $\Delta(X)$ satisfies the independence axiom ${ }^{13}$ if for all $p, q$ and $r$ in $\Delta(X)$ and for all $\alpha \in(0,1)$ such that $p \succeq q \Leftrightarrow \alpha p+(1-\alpha) r \succeq \alpha q+(1-\alpha) r$. Let $u$ represent the relation $\succeq$ on $\Delta(X)$, then we can express the independence in terms of $u$ as follows. For every $p, q, r$ in $\Delta(X)$ and for all $\alpha \in(0,1)$, we have

$$
\begin{gathered}
u(p, q) \geq u(q, p) \Leftrightarrow \\
u(\alpha p+(1-\alpha) r, \alpha q+(1-\alpha) r) \geq u(\alpha q+(1-\alpha) r, \alpha p+(1-\alpha) r) .
\end{gathered}
$$

If, in addition, the relation $\succeq$ satisfies SSB utility then $u$ is $\Delta$-bilinear by Proposition 8 and it implies that

$$
\begin{gathered}
u(p, q) \geq u(q, p) \Leftrightarrow \\
\alpha[u(p, q)-u(q, p)]+(1-\alpha)[u(p, r)-u(r, p)+u(r, q)-u(q, r)] \geq 0 .
\end{gathered}
$$

The following lemma will be useful for the proposition that follows afterwards. The lemma illustrates that the payoff function of a two-person symmetric game satisfies a kind of triangular equality in pure strategies if and only if it satisfies the similar triangular equality property in mixed strategies.

Lemma 1. Let $(\Delta(X), u)$ be a two-person symmetric game. For all $x, y, z$ in $X, u(x, y)+u(y, z)+u(z, x)=u(x, z)+u(z, y)+u(y, x)$ if and only if

$$
u(p, q)+u(q, r)+u(r, p)=u(p, r)+u(r, q)+u(q, p)
$$

for all $p, q, r$ in $\Delta X$.

\footnotetext{
${ }^{11}$ See Monderer and Shapley (1996) for more details.

${ }^{12}$ Note that the last equation is expressed in terms of the payoff function of player 1.

${ }^{13}$ Axiom of vN-M in the form of Jensen (1967). Actually, for our purpose it is enough to use a weaker independence axiom with $\alpha=\frac{1}{2}$. However, we use the most known version since SSB utility axioms together with weak independence imply the independence axiom. See Fishburn (1982) for details.
} 
Proof. ' $\Rightarrow$ ': By bilinearity of $u$, we have $u(p, q)=u_{p, q}=\sum_{x, y \in X} p_{x} q_{y} u_{x, y}$.

Substitute $u_{x, y}$ with $u_{x, z}+u_{z, y}+u_{y, x}-u_{y, z}-u_{z, x}$. Then for all $z \in X$, we have

$$
\begin{aligned}
& u_{p, q}=\sum_{x, y \in X} p_{x} q_{y}\left(u_{x, z}+u_{z, y}+u_{y, x}-u_{y, z}-u_{z, x}\right) \\
& =\sum_{x, y \in X} p_{x} q_{y} u_{x, z}+\sum_{x, y \in X} p_{x} q_{y} u_{z, y}+\sum_{x, y \in X} p_{x} q_{y} u_{y, x}-\sum_{x, y \in X} p_{x} q_{y} u_{y, z}-\sum_{x, y \in X} p_{x} q_{y} u_{z, x} \\
& \stackrel{(1)}{=} \sum_{x \in X} p_{x} u_{x, z} \sum_{y \in X} q_{y}+\sum_{x \in X} p_{x} \sum_{y \in X} q_{y} u_{z, y}+u_{q, p}-\sum_{x \in X} p_{x} \sum_{y \in X} q_{y} u_{y, z}-\sum_{x \in X} p_{x} u_{z, x} \sum_{y \in X} q_{y} \\
& \stackrel{(2)}{=} \sum_{x \in X} p_{x} u_{x, z}+\sum_{y \in X} q_{y} u_{z, y}+u_{q, p}-\sum_{y \in X} q_{y} u_{y, z}-\sum_{x \in X} p_{x} u_{z, x} \\
& \stackrel{(3)}{=} \sum_{x \in X}\left(p_{x}-q_{x}\right) u_{x, z}+\sum_{x \in X}\left(q_{x}-p_{x}\right) u_{z, x}+u_{q, p} . \\
& \text { We obtain (1) by rearranging the sums. Then, we obtain (2) by setting }
\end{aligned}
$$$$
\sum_{x \in X} p_{x}=1 \text {. Finally, by rearranging the index we obtain (3). Following the }
$$
same steps as above, we have the following equations,

$u_{q, r} \stackrel{(4)}{=} \sum_{x \in X}\left(q_{x}-r_{x}\right) u_{x, z}+\sum_{x \in X}\left(r_{x}-q_{x}\right) u_{z, x}+u_{r, q}$,

$u_{r, p} \stackrel{(5)}{=} \sum_{x \in X}\left(r_{x}-p_{x}\right) u_{x, z}+\sum_{x \in X}\left(p_{x}-r_{x}\right) u_{z, x}+u_{r, q}$.

Summing (3), (4) and (5) up yields $u_{p, q}+u_{q, r}+u_{r, p}=u_{p, r}+u_{r, q}+u_{q, p}$. '६': Trivial.

The following remark will be useful in the proof of the next proposition.

Remark 2. Given $(X, v)$, the following equations are equivalent for all $x, y, z, w$ in $X$.

$$
\begin{gathered}
v(x, y)+v(z, w)=v(x, w)+v(z, y) \\
v(x, y)=v(x, z)+v(z, y)
\end{gathered}
$$

Proof. ' $\Rightarrow$ ': Take $y=z$ then Equation 5.3 boils down to Equation 5.4. ' $\Leftarrow$ ': By Equation $5.4 v(x, y)=v(x, z)+v(z, y)$ and $v(z, w)=v(z, x)+$ $v(x, w)$. Summing up those two equations yield Equation 5.3.

The following proposition illustrates the relationship between a potential game and the independence axiom.

Proposition 10. Let $G=(\Delta(X), u)$ be a two-person symmetric game and let $(\Delta(X), \succeq)$ be its equivalent decision problem. The following statements are equivalent.

1. $\succeq$ satisfies the independence axiom. 
2. $G$ is a potential game.

3. $u(x, y)+u(y, z)+u(z, x)=u(x, z)+u(z, y)+u(y, x)$ for all $x, y, z$ in $X$.

Proof. (1) $\Rightarrow(3)$ : By Proposition 7, the equivalent decision problem of the game $(\Delta(X), u)$ satisfies $C, D$ and $S$. If, in addition, it satisfies the independence then by Proposition 1 of Fishburn (1982, p. 37) there exists a real valued function $\bar{u}$ such that $u(p, q)-u(q, p)=\bar{u}(p)-\bar{u}(q)$ for all $p, q$ in $\Delta(X)$. Then it follows for all $x, y, z$ in $X$ we have $u(x, y)-u(y, x)+u(y, z)-u(z, y)+$ $u(z, x)-u(x, z)=\bar{u}(x)-\bar{u}(y)+\bar{u}(y)-\bar{u}(z)+\bar{u}(z)-\bar{u}(x)=0$.

$(3) \Rightarrow(1)$ : By Lemma 1, condition (3) holds if and only if Equation 5.2 holds. Then, substituting $u(p, r)-u(r, p)+u(r, q)-u(q, r)$ with $u(p, q)-$ $u(q, p)$ reduces Equation 5.1 to $u(p, q) \geq u(q, p) \Leftrightarrow u(p, q) \geq u(q, p)$. Hence independence is trivially satisfied.

$(2) \Leftrightarrow(3)$ : We will use a sequence of equivalent statements for the demonstration. Define the two-person symmetric zero-sum game $(X, v)$ such that $v(x, y)=u(x, y)-u(y, x)$ for all $x, y, z$ in $X$. Then, by Theorem 20 of Duersch et al. (2012), $(X, u)$ is a potential game if and only if $(X, v)$ is a potential game. The latter holds if and only if Equation 5.3 holds according to Lemma 2.1 of Potters et al. (1999). Remark 2 concludes the proof since Equation 5.4 is equivalent to the statement in (3) by definition of $v$.

Corollary 3. A decision problem satisfies von Neumann-Morgenstern utility if and only if its equivalent symmetric game is a potential game.

It may be of interest to note the following relation between vN-M utility, Shapley value and potential games. Roth (1977) showed that the Shapley value of a player coincides with the vN-M utility function representing the preferences (satisfying some conditions) of the player over the positions in a game. ${ }^{14}$ Consider a decision problem whose set of alternatives consist of different positions in a game. If the decision maker has preferences satisfying the axioms of Roth (1977) over positions. Then, the equivalent game of the decision problem is a potential game if and only if the Shapley value (of the decision maker in the initial game) is the vN-M utility function representing the preferences in the extended decision problem.

Among the axioms of expected utility of $\mathrm{vN}-\mathrm{M}$, perhaps independence is the most attacked axiom partly due to its strong implications. One of

\footnotetext{
${ }^{14}$ The vN-M utility function should be normalized. See Roth (1977) for details.
} 
the best known example is the Allais paradox which is an illustration of the systematic violation of it. Here, we try to show the strength of the independence axiom by a different approach, that is, by examining the effect of imposing the independence axiom on a preference relation satisfying SSB utility. The next proposition gives a formula to obtain the number of linearly independent equations that must be satisfied in order for triangular equality condition to hold.

Proposition 11. Given a matrix $\left[u_{i j}\right]_{n \times n}$ with $n \geq 3$, let the property $(P)$ be such that for all $i, j$ and $k$ we have $u_{i j}+u_{j k}+u_{k i}=u_{i k}+u_{k j}+u_{j i}$ for $i, j, k=1,2, \ldots, n$. Then the number of linearly independent equations needed for $(P)$ to be satisfied is given by $\frac{(n-1)(n-2)}{2}$.

Proof. Let $E_{i j k}$ denote the equation $u_{i j}+u_{j k}+u_{k i}=u_{i k}+u_{k j}+u_{j i}$ and denote by $E$ the set of all these equations. We can restrict the attention to the case $i<j<k$ because the equations $E_{i j k}, E_{i k j}, E_{j i k}, E_{j k i}, E_{k j i}$ and $E_{k i j}$ represent the same equation. The sums do not change no matter at which node you start summing and whether you go first clockwise or counter clockwise, having the figure below in mind.

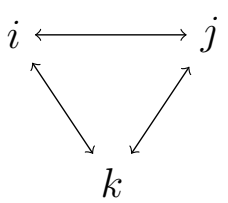

We show that $E^{\prime}=\left\{E_{1 l m} \mid 1<\ell<m\right\}$ is a basis for $E$. To see this, first notice that for any quadruple $i<\ell<k<m$, the equations $E_{i j k}, E_{i j m}$ and $E_{i k m}$ imply the equation $E_{j k m}$. From this it follows that any equation $E_{i j k}$ with $i \neq 1$ can be obtained from equations $E_{1 i j}, E_{1 i k}$ and $E_{1 j k}$. Left to show is that an equation in $E^{\prime}$ cannot be obtained as a linear combination of the other equations in $E^{\prime}$. By contraposition, suppose $E_{1 j k}$ is a linear combination of equations in $E^{\prime} \backslash\left\{E_{1 j k}\right\}$. Then, there exists at least one equation in $E^{\prime}$ which contains the number $u_{j k}$ (otherwise the $u_{j k}$ can never appear). But then, this equation must be $E_{1 j k}$, which is in contradiction with our supposition. The number of equations in $E^{\prime}$ is precisely the number given in the proposition.

Proposition 11 gives a formula to obtain the number of linearly independent equations that must be satisfied in order to pass from SSB utility to 
expected utility. Notice that the number $\frac{(n-1)(n-2)}{2}$ grows quadratically as the number of alternatives grows. This might be considered as an indication of the strength of the independence axiom.

Even though it weakens the independence axiom of vN-M utility, SSB utility theory is not problem-free either. For instance, Fishburn (1984, p. 139) mentions some of potentially disturbing implications of SSB utility in the case of cycling preferences as follows. Consider the decision problem $(\Delta(X), v)$ with SSB utility where $X=\{x, y, z\}$ and $x \succ y \succ z \succ x$. Suppose we replace $z$ by $z^{\prime}$ such that $z^{\prime}$ in $X^{\prime}=\left\{x, y, z^{\prime}\right\}$ does better than $z$ against $x$ and against $y$ but preferences still cycle, that is, $v\left(z^{\prime}, x\right)>v(z, x)>0$ and $v(y, z)>v\left(y, z^{\prime}\right)>0$. Let $p$ and $p^{\prime}$ be the maximal elements in $(\Delta(X), v)$ and in $\left(\Delta\left(X^{\prime}\right), v\right)$ respectively. Then it might happen that $p\left(z^{\prime}\right)<p(z)$. This is a bit unintuitive because $z^{\prime}$ is better than $z$ with respect to both $x$ and $y$. This situation, however, is a known phenomenon of mixed strategy Nash equilibria in games. Consider the equivalent two-person zero-sum game $(\Delta(X), v)$ of the decision problem. Everything else being equal, when we increase a particular payoff of a player, it sometimes becomes less likely to be played in the Nash equilibrium. For example, suppose that we increase the payoff of $v(z, x)$ from 1 to 2 and the payoff of $v(z, x)$ from -1 to $\frac{-1}{2}$ and that we rearrange the payoffs so that the rock-paper-scissors game in Figure 1 remains a symmetric zero-sum game. Then the probability of $z$ being played at the Nash equilibrium decreases from $\frac{1}{3}$ to $\frac{2}{7}$.

\section{Conclusion}

In this paper, we have showed that there is an equivalence between the class of two-person symmetric games and the class of one-person decision problems in which the preferences are complete but not necessarily transitive. By making use of this, we illustrated some equivalences between the existing notions in games and in decision problems. For instance, we showed that a two-person symmetric zero-sum game can be extended to its von Neumann-Morgenstern mixed extension if and only if the extended decision problem satisfies the SSB utility axioms. In zero-sum symmetric games, we also showed that a strategy is an optimal strategy if and only if it is a maximal element in the equivalent decision problem of the game. Regarding two-person symmetric games, we proved that an optimal threat strategy and a maximal element of the equivalent decision problem of the game coincide. 
In addition, we demonstrated that a two-person symmetric game is a potential game if and only if its equivalent decision problem satisfies von Neumann-Morgenstern utility. Accordingly, we provide a formula for the number of linearly independent equations that must be satisfied in order to pass from SSB utility to von Neumann-Morgenstern utility. This number grows quadratically as the number of alternatives increase. We discuss that this may be interpreted as an alternative way to show the strength of the independence axiom.

\section{References}

Debreu, G. (1954). Representation of a preference ordering by a numerical function. In R. Thrall, C. Davis, and R. Coombs (Eds.), Decision Processes, pp. 159-165. John Wiley.

Duersch, P., J. Oechssler, and B. C. Schipper (2012). Pure strategy equilibria in symmetric two-player zero-sum games. International Journal of Game Theory 41, 553-564.

Fishburn, P. C. (1982). Nontransitive measurable utility. Journal of Mathematical Psychology 26(1), 31-67.

Fishburn, P. C. (1984). Dominance in SSB utility theory. Journal of Economic Theory 34(1), 130-148.

Fishburn, P. C. (1988). Nonlinear preference and utility theory. Johns Hopkins University Press, Baltimore.

Fudenberg, D. and D. K. Levine (2006). A dual-self model of impulse control. The American Economic Review 96(5), pp. 1449-1476.

Jensen, N. E. (1967). An introduction to Bernoullian utility theory: I. utility functions. The Swedish Journal of Economics 69(3), 163-183.

Monderer, D. and L. S. Shapley (1996). Potential games. Games and Economic Behavior 14(1), $124-143$.

Nash, J. (1951). Non-cooperative games. Annals of Mathematics 54(2), pp. 286-295. 
Nash, J. (1953). Two-person cooperative games. Econometrica 21(1), pp. $128-140$.

Owen, G. (1968). Game Theory. Academic Press.

Potters, J., T. Raghavan, and S. Tijs (1999). Pure Equilibrium Strategies for Stochastic Games Via Potential Functions. UB Nijmegen [Host].

Rosenthal, R. W. (1973). A class of games possessing pure-strategy Nash equilibria. International Journal of Game Theory 2(1), 65-67.

Rosenthal, R. W. (1981). Games of perfect information, predatory pricing and the chain-store paradox. Journal of Economic Theory 25(1), 92 - 100.

Roth, A. E. (1977). The Shapley value as a von Neumann-Morgenstern utility. Econometrica 45(3), 657-664.

Schaffer, M. E. (1988). Evolutionarily stable strategies for a finite population and a variable contest size. Journal of Theoretical Biology 132(4), 469-478.

Tullock, G. (1964). The irrationality of intransitivity. Oxford Economic Papers, New Series 16, 401-406.

von Neumann, J. (1928). Zur Theorie der Gesellschaftsspiele. Mathematische Annalen 100, 295-320.

von Neumann, J. and O. Morgenstern (1944). Theory of Games and Economic Behavior (1953, Third ed.). Princeton University Press. 